\title{
Music Preference in Degus (Octodon degus): Analysis with Chilean Folk Music
}

\author{
Shigeru Watanabe ${ }^{1 *}$, Katharina Braun ${ }^{2}$, Maria Mensch ${ }^{2}$, and Henning Scheich ${ }^{3}$ \\ ${ }^{1}$ Keio University, Minato-ku, Mita 2-15-45,Tokyo Japan \\ ${ }^{2}$ Otto Von Guericke University, Magdeburg, Germany \\ ${ }^{3}$ Leibniz Institute for Neurobiology \\ *Corresponding author (Email: swat@ flet.keio.ac.jp)
}

Citation - Watanabe, S., Braun, K., Mensch, M., \& Scheich, H. (2018). Music preference in degus (Octodon degus): Analysis with Chilean folk music. Animal Behavior and Cognition, 5(2), 201-208. https://doi.org/10.26451/abc.05.02.02.2018

\begin{abstract}
Most nonhuman animals do not show selective preference for types of music, but researchers have typically employed only Western classical music in such studies. Thus, there has been bias in music choice. Degus (Octodon degus), originally from the mountain areas of Chile, have highly developed vocal communication. Here, we examined music preference of degus using not only Western classical music (music composed by Bach and Stravinsky), but also South American folk music (Chilean and Peruvian). The degus preferred the South American music to the Western classical music but did not show selective preference between the two Western classical music choices. Furthermore, the degus preferred the Chilean to the Peruvian music to some extent. In the second experiment, we examined preference for music vs. silence. Degus overall showed a preference for Chilean music over silence, but preferred silence over Western music. The present results indicate that the previous negative data for musical preference in nonhuman animals may be due to biased music selection (Krause, 2012). Our results suggest the possibility that the soundscape of an environment influences folk music created by native peoples living there and the auditory preference of other resident animals there.
\end{abstract}

Keywords - Degu, Music preference, Chilean music, Reinforcement

Hearing music often causes a pleasurable experience in humans, but nonhuman animals do not display such pleasure (see review by Watanabe 2012, 2015). Therefore, the reinforcing property (or pleasure) of music has been concluded to be a human-specific trait (McDermott \& Hauser, 2004). We have, however, demonstrated that Java sparrows (Lonchura oryzivora) show a selective preference for particular music (Watanabe \& Nemoto, 1998). Although the reinforcing property of music has not been thoroughly examined, humans and songbirds (Java sparrows) appear to be exceptional species for showing preferences for particular types of music (Gess, 2007). Most of the relevant published studies have failed to demonstrate any reinforcing effect of music in primates. Chimpanzees (Pan troglodytes, Howell, Schwandt, Fritz, Roeder, \& Nelson, 2003), gorillas (Gorilla gorilla gorilla, Wells, Coleman, \& Challis, 2006), the common marmoset (Callithrix jacchus) and cotton-top tamarin (Saguinus oedipus, McDermott \& Hauser, 2004, 2007), rats (Rattus norvegicus, Otsuka, Yanagi, \& Watanabe, 2009), pigeons (Columba livia, Watanabe, Suzuki, \& Yamazaki, 2009) and goldfish (Carassius auratus, Shinozuka, Ono, \& Watanabe, 2013) did not show musical preference. 
One common bias in the design of music preference experiments is the choice of music. Nearly all previous studies, including our own, have presented animals with Western classical music. Ritvo and MacDonald (2016) examined musical preference in the Sumatran orangutan (Pongo abelli). They presented orangutans with different kinds of music, including non-Western music (Mongolian Tuva throat singing). However, all of them showed a preference for silence over music or showed no clear preference. Mingle, Eppley, Campbell, Hall, and de Waal (2014) presented traditional African music to chimpanzees and observed that the animals moved closer to the sound source when African music was broadcast. These results suggest the possibility of preference for native folk music in the animals' habitat over Western (non-habitat) music in nonhuman animals. Snowdon and Teie (2010) produced music composed with characteristics of animal vocalization and observed that tamarins preferred such music over human music. Snowdon, Teie, and Savage (2015) created species-appropriate music and found that cats preferred the music over human-appropriate music.

The degu, a so-called "singing rat" of the Andes, is a rodent that originates in the mountainous areas of Chile. They have more than 15 sounds (Long, 2007). This species is considered suitable for musical preference studies for several reasons. First, they have highly developed auditory communication, which makes auditory stimuli important information for them. Second, their hearing range is similar to that of humans (Braun, Kremz, Wetzel, Wagner, \& Poeggel, 2003), which suggests that human-made music would be suitable for their auditory sensation. Here, we tested the music preference of degus using Western music and South American folk music.

\section{Experiment 1: Preference Among Western, Chilean, and Peruvian Music}

\section{Method}

Subjects. The 42 male degus (Octogon degus) used in this study were bred in our colony (Institute of Biology, Otto von Guericke University, Magdeburg). Animals were group-caged in wire cages $(51 \times 42 \times 68 \mathrm{~cm})$ in air-conditioned rooms with an average temperature of $22^{\circ} \mathrm{C}$, under artificial light/dark cycle $(12 \mathrm{~h} / 12 \mathrm{~h})$. Fresh water, food pellets and vegetables were available ad libitum. The experiment was carried out across five years (2013 - 2017). The age of animals ranged from one to three years. We used 12 animals for the preference tests with Western music, ten animals for the preference tests with Chilean and Western music, ten animals for the preference tests with Chilean, Peruvian and Western music, and ten animals for the music vs. silence experiment. We used experimentally naïve animals in each year. The experimental protocols were conducted according to the German guidelines for the care and the use of animals in laboratory research ( $\$ 8$, Abs.1, 25.05.1998), and all experiments were performed in accordance with the European Communities Council Directive of November 1986 (86/609/EEC).

Apparatus. The experimental chamber was a $120 \times 39 \times 70 \mathrm{~cm}$ white box (Figure 1) with separate speakers at each end. Two photo-sensors connected to each speaker (one at $3 \mathrm{~cm}$ and the other at $16 \mathrm{~cm}$ from the speaker) were affixed to the sidewalls of the chamber. Two music sticks (Intenso MP3 player) continuously played music but the relay circuit connected to the photo-sensors (either one at $3 \mathrm{~cm}$ or one at $16 \mathrm{~cm}$ ) could switch on the output of the music sticks to a speaker only when the animal crossed one of the photo-sensors paths. A start box $(31 \times 31 \times 31 \mathrm{~cm})$ with a guillotine door was connected to the chamber. Behavior of the subjects was recorded through a video camera (WV-CP480, Panasonic) above the experimental chamber and analyzed after the test using the EthoVision software. The chamber was divided into three areas, namely the left, central and right areas. The start box opened into the central area. The left and right areas (20 cm from the each speaker) were the approximate space in which the subjects could hear the music.

Stimuli. The two Western classical music pieces used were the Toccata and Fugue in D minor (BWV 565) (J. S. Bach), which is played by pipe organ, and The Rite of Spring (I. Stravinsky), which is played by orchestra. The Chilean music was "Gracias a la Vida" played with charango (stringed instrument) by Ethnophonic Ensemble and the Peruvian music was "El condor pasa" played with quena 
(traditional flute). The reason that we selected these pieces by Bach and Stravinsky is that we had used them in previous animal experiments (Otsuka et al, 2009; Shinozuka et al., 2013; Watanabe et al., 2009; Watanabe \& Nemoto, 1998), thus allowing us to compare the results with our previous experiments. As for the Chilean and Peruvian music, we selected these particular pieces for two reasons. First, the music is played with folk instruments, and not Western instruments. Second, in searching for Chilean and Peruvian music on the Internet, these pieces were often retrieved, suggesting that they are relatively common pieces. Because the length of the music pieces differed markedly due to the different types of music, we recorded $20 \mathrm{~s}$ clips from each piece into a music stick MP3 player. These sounds were repeatedly played, but animals could hear the sound only when they stayed in the music areas and crossed the sensors. The loudness at the photo-sensor was 58-70 dB for the Toccata and Fugue, 59-71 dB for The Rite of Spring, 60-69 dB for "Gracias a la Vida" and 60-70 dB for "El condor pasa." Thus, there were no systematic differences in loudness between the music types.

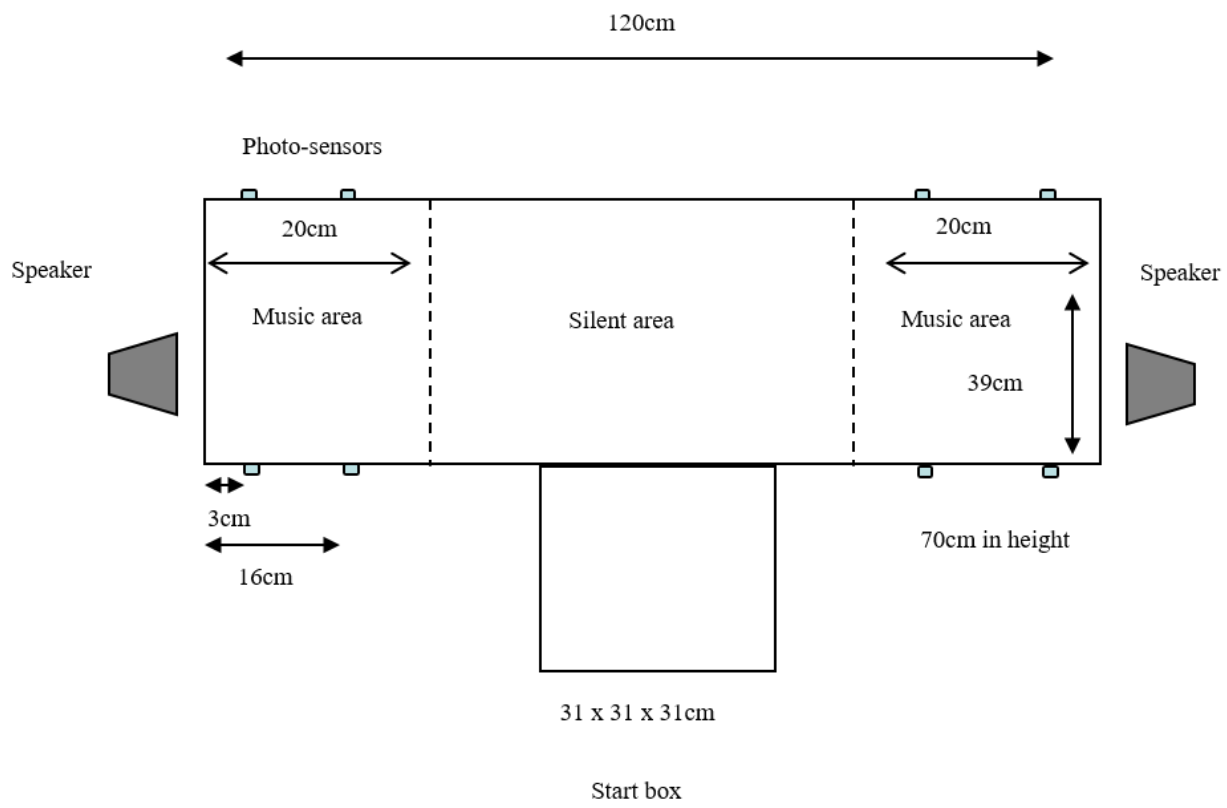

Figure 1. Schematic of the preference test. The smaller box is the start box. The dashed lines indicate the area in which the animals could hear the music.

Procedure. The procedure consisted of adaptation and preference test phases. During the adaptation phase the animals were placed in a start box for two minutes then released into the test chamber and allowed to move freely around the chamber for five minutes with no auditory stimuli.

Testing started on the day after adaptation. The subjects were placed in the start box for 2 min and then released into the chamber. They were allowed to move around the chamber for 10 min, during which the two speakers presented them with the two different types of music whenever they activated the photo-sensors. For half of the subjects, one music piece was presented from the left speaker and the other music piece from the right speaker. This orientation was reversed for the remaining subjects. We carried out preference testing twice a day, at 9:00 in the morning and at 14:00 in the afternoon. The music at each speaker was reversed in the afternoon session. Thus, each animal received a particular stimulus from both the right and left speakers in one day to neutralize possible side preference.

Statistical Analysis. We used time spent (s) within $20 \mathrm{~cm}$ from each speaker as an index of preference and employed two-tailed paired $t$-tests to assess the results from each preference experiment. In the South American folk and Western music comparison, data from the South American vs. Bach and the South American vs. Stravinsky tests were integrated. The difference in the time spent in each music area, $20 \mathrm{~cm}$ from the end wall, from the chance level (i.e., $100 \mathrm{~s}$ ) was also examined using a one-sample $t$ test. 


\section{Results}

Figure 2A shows the results of the Western music preference test. The subjects were placed in a rectangular experimental chamber in which a speaker was fixed at each end wall (see Figure 1). Photosensors activated each speaker to broadcast one of the two music choices when the animal was in close proximity. We measured the time spent near each speaker during a $10 \mathrm{~min}$ period to detect preference between the two music choices. The first test compared two different Western music pieces, Toccata and Fugue in D minor (BWV 565) by J. S. Bach and The Rite of Spring by I. Stravinsky. As shown in Figure $2 \mathrm{~A}$, the subjects did not show a particular preference for either classical style. The two-tailed paired $t$-test showed no significant difference in time spent at the two sites $(t(11)=0.11, p=.91,95 \%$ CI [-64.93, 58.53]). One animal consistently stayed more than $60 \%$ of the total time in the music areas at the right music area. A one-sample $t$-test revealed no significant difference from the chance level in the time spent in the Bach area $(t(11)=0.49, p=.64,95 \%$ CI 95\% $[-52.24,81.54])$ or in the Stravinsky area $(t(11)=$ $0.59, p=.56,95 \%$ CI $[-48.27,83.96])$. Thus, degus did not show selective preference for either Western music style.
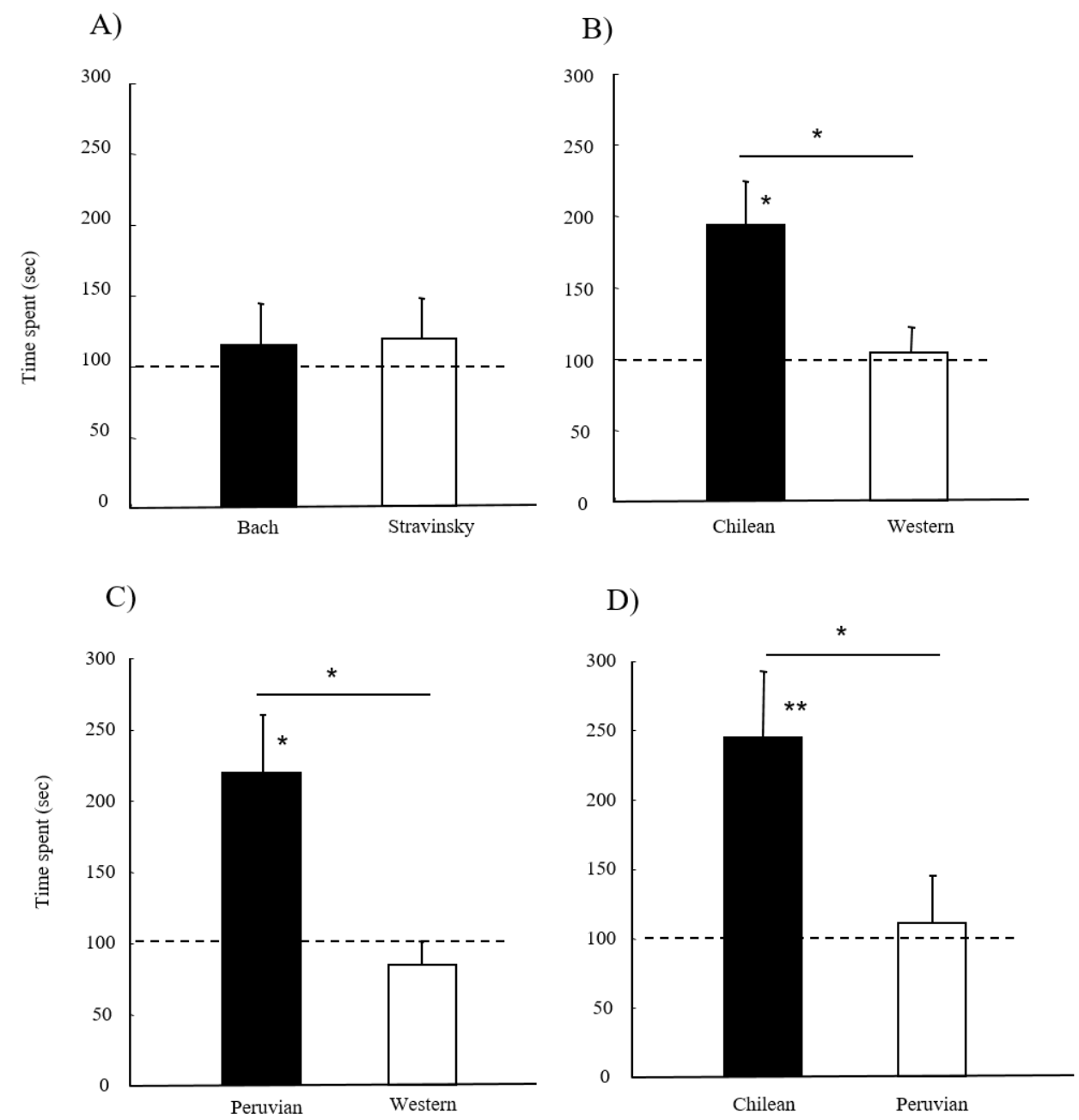

Figure 2. Musical preference in Degus (Experiment 1). A) Degus did not show selective preference for Bach or Stravinsky. Degus preferred Chilean folk music to the Western music (B) and also preferred Peruvian folk music to the Western music (C). D) They also preferred the Chilean to the Peruvian music. The broken line indicates chance level time spent in each music area. Vertical bars indicate SE. *p<.05,**p<.01. 
The second test examined preference between the Chilean folk instrumental music piece "Gracias a la Vida" and each of the two Western music pieces (Figure 2B). There was a significant difference in time spent near the Chilean and either of the Western music pieces (two-tailed paired $t$-test, $t(18)=2.14$, $p=.04,95 \%$ CI $[1.51,177.56])$. There was a significant difference from the chance level in time spent in the Chilean area $(t(18)=2.89, p=.01,95 \%$ CI $[25.50,160.74])$, but no significant difference in time spent in the Western area $(t(18)=0.19, p=.85,95 \%$ CI $[-34.50,41.67])$. Thus, the degus displayed preference for Chilean folk music over Western classical music.

Figure $2 \mathrm{C}$ shows the results of the third test in which we compared the Peruvian folk instrumental music piece "El condor pasa" to each of the two Western music pieces. There was a significant difference in time spent near the Peruvian music vs. the Western music pieces (two-tailed paired, $t$-test $t(18)=2.60$, $p=.02,95 \%$ CI $[26.03,244.06])$. There was a significant difference in time spent in the Peruvian area from chance $(t(18)=2.76, p=.02,95 \%$ CI $[28.54,209.68])$, but no significant difference for time spent in the Western area $(t(18)=0.92, p=.37,95 \%$ CI $[-52.27,20.39])$. Thus, degus displayed preference for Peruvian music over Western music.

The last test compared the response to the Peruvian and Chilean Instrumental folk music used in the previous test (Figure 2D). There was a significant difference in time spent near the Chilean and Peruvian music (two-tailed paired $t$-test, $t(10)=2.48, p=.03,95 \%$ CI $[13.85,254.25]$ ). There was a significant difference from the chance level in time spent in the Chilean area $(t(9)=3.41, p=.01,95 \% \mathrm{CI}$ $[37.18,183.95])$ but no significant difference in time spent in the Peruvian area $(t(9)=0.64, p=.54,95 \%$ CI $[-54.54,97.86])$. Thus, degus appeared to display preference for Chilean music over Peruvian music to some extent.

\section{Experiment 2: Preference Between Music and Silence}

Experiment 1 demonstrated degus' selective preference for different types of music, revealing that they stayed in the Chilean or Peruvian music areas longer than they did in the Western music areas. In addition, they stayed at the Chilean or Peruvian music areas longer than would be expected by chance, suggesting that they preferred this music rather than that they stayed in those areas in order to escape from the Western music. To confirm this preference, Experiment 2 compared time spent in a music area with that in an area without music. The same apparatus and music as used in Experiment 1 were employed in this study. Subjects were ten experimentally naïve male degus less than one year old. The procedure was identical to that in Experiment 1, except that one of the music areas did not present music. Because there were four different music pieces, each subject underwent eight tests.

Results were analyzed in three different ways. The first is comparison of time spent in the music area with that in the area without music with a paired $t$-test. The time spent in the music area was compared with the chance level staying time (i.e., $100 \mathrm{~s}$ ) using a one-sample $t$-test. Finally, time spent in each music area was analyzed by one-way ANOVA and Shaffer's modified sequential rejective Bonferroni procedure.

\section{Results}

Figure 3 presents the results. There was a significant difference in time spent between the Chilean music and silence areas $(t(9)=2.80, p=.02,95 \% \mathrm{CI}[11.60,110.06])$, between the Bach and silence areas $(t(9)=2.80, p<.02,95 \%$ CI $[11.60,110.06])$, and between the Stravinsky and silence areas $(t(9)=2.84, p$ $=.02,95 \%$ CI $[-98.94,-11.28])$. However, there was no significant difference between the Peruvian music and silence areas $(t(9)=1.25, p=.24,95 \%$ CI $[-48.60,168.95])$. The degus preferred the Chilean music to silence, but preferred silence to Bach or Stravinsky. They also showed no selective preference for Peruvian music or silence.

When comparing time spent with the chance level (100 s), we found a significant difference from the chance level in time spent in the Chilean music area $(t(9)=3.49, p=.007,95 \%$ CI [17.11, 79.95]), Bach area $(t(9)=-4.47, p=.002,95 \%$ CI $[-59.10,-25.74])$, and Peruvian area $(t(9)=-2.481, p=.03,95 \%$ 


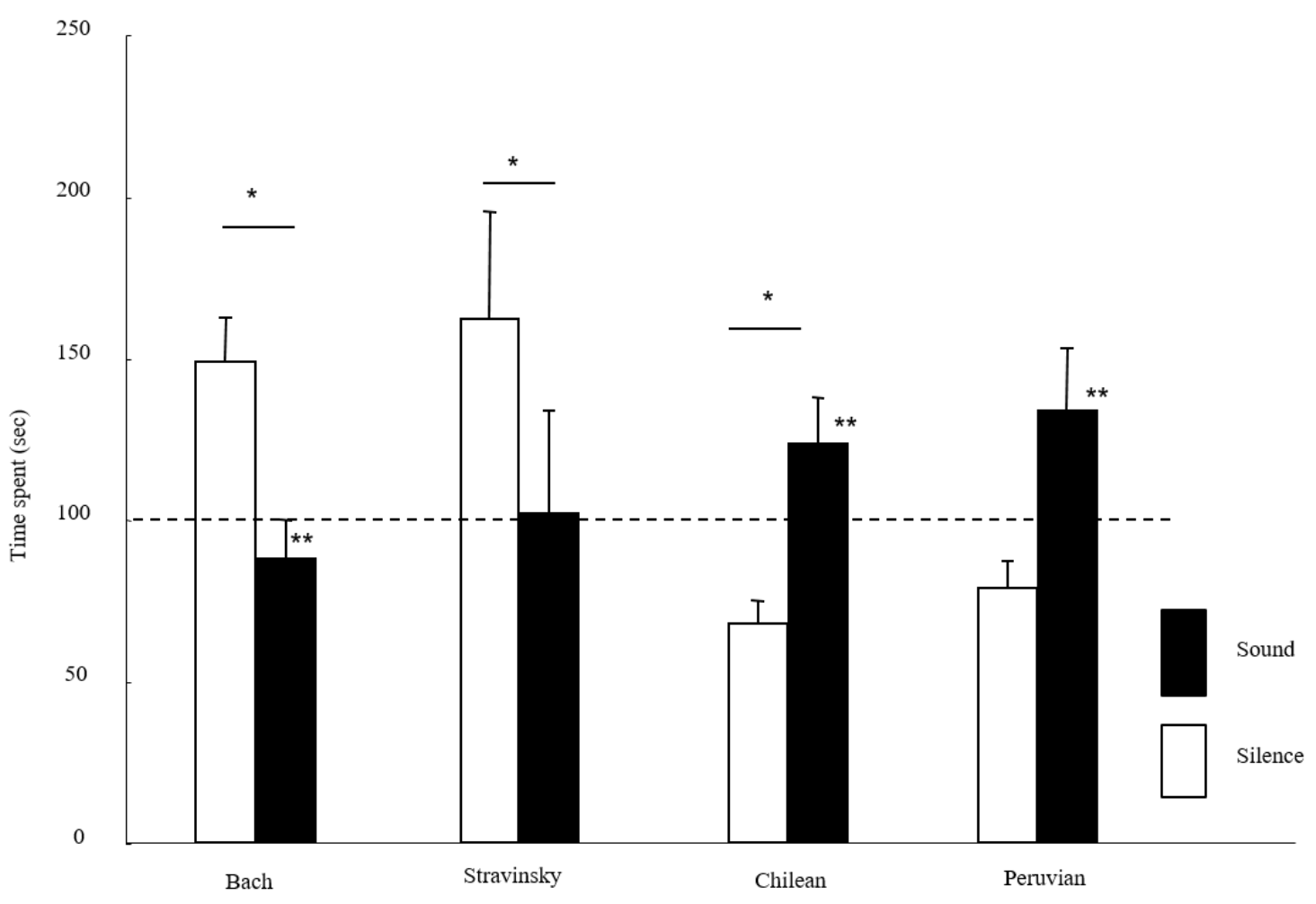

Figure 3. Music preference to silence (Experiment 2). Dark bars indicate time spent in music area and open bars those in the silence area. Broken line indicates chance level time spent in music area. Vertical bars indicate SE. * $p<.05, * * p<.01$.

CI $[-40.03,-16.06])$. However, time spent in the Stravinsky area did not significantly differ from the chance level $(t(9)=-1.87, p=.09,95 \%$ CI $[-12.76,136.89])$.

One-way ANOVA of time spent in the music areas revealed a significant effect of the music $(F$ $(3,39)=6.45, p=.002)$. Shaffer's modified Bonferroni procedure gave a significant difference between Chilean and Bach $(t(9)=6.49, p=.0001)$ or Stravinsky $(t(9)=4.43, p=.002)$, and between Peruvian and Bach $(t(9)=2.45, p=.04)$ or between Peruvian and Stravinsky $(t(9)=2.74, p=.02)$. There was no significant difference between Chilean and Peruvian $(t(9)=0.37, p=.72$ ) or between Bach and Stravinsky $(t(9)=.87, p=.41)$. Thus, the degus preferred Chilean and Peruvian music to the Western music.

\section{Discussion}

The present results are the first demonstration of a music style preference in degus. The degus prefer South American folk instrumental music to Western music. They did not show selective preference for either Western music style, but showed preference for Chilean music over Peruvian.

Because the degus preferred the Chilean music not only to the Western music but also to the silence, it seems clear that they actively preferred the Chilean music and that their selection was not the result of trying to escape from the Western music, which had an aversive effect on them. The lack of a preference for Western music in degus accords with previous research on other animal species, with the exception of songbirds. It is interesting that the degus avoided the Western music. In a previous experiment with Java sparrows, some birds showed an avoidance of Stravinsky but a preference for Bach (Watanabe \& Nemoto, 1998). They also preferred silence over Stravinsky. Chimpanzees preferred African and Indian music (Mingle et al., 2014), but did not show a preference for Japanese music. It is, however, premature to conclude that these data reveal their general preferences. Furthermore, Sumatran 
orangutans did not show a preference for Mongolian Tuva throat singing over Western music (Ritvo \& MacDonald, 2016), but because orangutans are not Mongolian animals and the researchers did not examine Sumatran music, it remains unclear whether orangutans prefer native folk music in Sumatra over Western music.

We have to point out three possible factors in the present experiments. First, we used degus of different ages (ranging from one to three years) but did not find any systematic differences according to age. Second, all of the subjects in the present study were male. Although both males and females produced a variety of sounds and there are no sex differences in the role of singer and receiver (a phenomenon found in songbirds), it is still possible that degus show sexually dimorphic vocalization. For example, Wied's black-tufted-ear marmosets (Callithrix kuhlii) showed sex-dependent variations in vocalization (Smith, Birnie, Lane, \& French, 2009). Thus, it is possible that female degus showed different musical preferences. Third, side preference might affect the results. Consistent preference for one side might result in no apparent preference for music. In one sense, strong side preference indicates absence of music preference or overshadowing weak music preference by the side preference. We found only one subject showing a consistent side preference in Experiment 1 and no subjects showed more than $60 \%$ side preference in Experiment 2. Thus, the side preference did not affect the results.

Because music is a quite complex stimulus, the present experiments cannot clarify which aspect of the music has reinforcing properties. It is also premature to conclude that the degus prefer Chilean music "in general" or that they dislike Western music "in general," but the present results display the presence of musical preference in degus. One possible explanation for the preference of degus for South American folk music is based on their complex auditory communication. However, in contrast to Java sparrows that also have complex auditory communication, the degus' preference appears to be restricted to the South American music as far as we know.

Physical properties of the environment produce a particular soundscape (biophony and geophony). Development of folk music by native peoples may be influenced by the sound scape of their living area, and nonhuman animals living in that area may have similarly developed preference for the particular acoustic stimuli based on the sound scape. Biomusicologists investigate the links between acoustic configurations in nature and the evolution of human music (Wallin, Bjorn, \& Brown, 2001). For example, similarities between the sounds of the Dzanga-Santgha rain forest to music of the Ba'Aka people (Sarno, 1996), as well as the influence of the rhythms and melodies of rain striking vegetation and traditional music of the Yamanomi (Krause, 2012) are pointed out. Although the explanation of degus' musical preference in terms of biophony is speculative, the present study may suggest that some animals may prefer human music produced by people who share the biophony and geophony with such animals.

\section{Acknowledgements}

This research was supported by Japan Society for the Promotion of Science (25118001), German Science Foundation DFG (SFB 779) and the German-Israeli Science Foundation (\#1114-101.4/2010).

\section{References}

Braun, K., Kremz, P., Wetze, W., Wagner, T., \& Poeggel, G. (2003). Influence of parental deprivation on the behavioural development in Octodon degus: Modulation by maternal vocalizations. Developmental Psychobiology, 42, 237-245.

Gess, A. (2007). Birds like music, too. Science, 317, 1864.

Howell, S., Schwandt, M., Fritz, J., Roeder, E., \& Nelson, C. (2003). A stereo music system as environment enrichment for captive chimpanzees. Laboratory Animals, 32, 31-36.

Krause, B. (2012). The great animal orchestra. New York City, USA Little Brown and Company.

Long, C. V. (2007). Vocalisations of the degu (Octodon degus), a social caviomorph rodent. Bioacoustics, 16, 223244.

McDermott, J., \& Hauser, M. D. (2004). Are consonant intervals music to their ear? Spontaneous acoustic preferences in nonhuman primates. Cognition, 94, B11-B21. 
McDermott, J., \& Hauser, M. D. (2007). Nonhuman primates prefer slow tempos but dislike music overall. Cognition, 104, 654-668.

Mingle, M. E., Eppley, T. M., Campbell, M., Hall, K, \& de Waal, F. M. M. (2014). Chimpanzees prefer African and Indian Music over silence. Journal of Experimental Psychology: Animal Learning and Cognition, 4, 502505.

Otsuka, Y., Yanagi, J., \& Watanabe, S. (2009). Discrimination and reinforcing stimulus properties of music for rats. Behavioural Processes, 80, 121-127.

Ritvo, S. E., \& MacDonald, S. E. (2016). Music as enrichment for Sumatran orangutans (Pongo abelii). Journal of Zoo and Aquarium Researches, 4, 1-8.

Sarno, L. (1996). Bayaka: The extraordinary music of the Babenzele Pygmies. New York City, USA: Ellipsis Arts.

Shinozuka, K., Ono, H., \& Watanabe, S. (2013). Reinforcing and discriminative stimulus properties of music in goldfish. Behavioural Processes, 99, 26-33.

Smith, A. S., Birnie, A. K., Lane, K. R., \& French, J. A. (2009). Production and perception of sex differences in vocalizations of Wied's black-tufted-ear marmosets (Callithrix kuhlii). American Journal of Primatology, 71, 324-332.

Snowdon, C. T., \& Teie, D. (2010). Affective responses in tamarins elicited by species-specific music. Biology Letters, 6, 30-32.

Snowdon, C. T., Teie, D., \& Savage, M. E. (2015). Cats prefer species appropriate music. Applied Animal Behaviour Science, 166, 106-111.

Wallin, N., Bjorn, M., \& Brown, S. (2001). The origins of music. Cambridge, MA, MIT Press.

Watanabe, S. (2012). Animal aesthetics from the perspective of comparative cognition. In S. Watanabe \& S. Kuczaj (Eds.), Emotions of animals and humans (p. 129-164). Tokyo, Japan: Springer.

Watanabe S. (2015). Aesthetics and reinforcement: A behavioral approach to aesthetics. In T. Hoquet (Ed.), Current perspectives on sexual selection: What's left after Darwin (pp. 289-307). Dordrecht: Springer.

Watanabe, S., \& Nemoto, M., (1998). Reinforcing property of music in Java sparrows (Padda oryzivora). Behavioural Processes, 43, 211-218.

Watanabe, S., Suzuki, T., \& Yamazaki, Y. (2009). Reinforcing property of music for nonhuman animals: Analysis with pigeons. Philosophy, 121, 1-21.

Wells, D. L., Coleman, D., \& Challis, M. G. A. (2006). A note on the effect of auditory stimulation on the behavior and welfare of zoo-housed gorillas. Applied Animal Behavior Science, 100, 327-332. 